\title{
Revascularização total do miocárdio sem circulação extracorpórea: cinco anos de experiência
}

\author{
Total myocardium revascularization without extracorporeal circulation: five-year experience
}

Rodrigo MILANI, Paulo BROFMAN, Alexandre VARELA, José Augusto MOUTINHO, Maximiliano GUIMARÃES, Rafael PANTAROLLI, Laura BARBOSA, Alexandre BARBOSA, Glauco PINA, Francisco MAIA

RBCCV 44205-429

\section{Resumo}

Objetivo: Avaliar os resultados imediatos da operação para revascularização do miocárdio sem circulação extracorpórea, analisando-se complicações e mortalidade.

Método: Foram submetidos à operação para revascularização do miocárdio sem circulação extracorpórea 1440 pacientes. A técnica operatória consistiu em oclusão proximal da artéria abordada, aplicação do ponto de LIMA na deflexão pericárdica posterior e estabilização da artéria alvo com estabilizador de sucção. As anastomoses distais foram feitas inicialmente.

Resultados: Entre os pacientes avaliados, 924 eram ao sexo masculino, com idade média de $63,12 \pm 8,76$ anos. A fração de ejeção era normal em 749 pacientes. Setecentos e quarenta $(51,4 \%)$ pacientes tinham antecedente de infarto do miocárdio. Seiscentos e oitenta e sete $(\mathbf{4 7 , 6 \% )}$ pacientes encontravamse em classe funcional III ou IV. O EuroSCORE médio foi de $4,93 \pm 3,32$. A média de anastomoses distais foi de $3,12 \pm 1,23$ por paciente. Mil cento e setenta e três $(81,5 \%)$ pacientes permaneceram menos de 12 horas em ventilação mecânica, sendo que destes, $888(61,7 \%)$ permaneceram menos de 6 horas entubados. A permanência em UTI foi de uma noite em $330(22,8 \%)$ pacientes e de duas noites em $930(64,6 \%)$. Cento e oitenta e dois $(12,4 \%)$ pacientes permaneceram três ou mais noites na UTI. Quanto às complicações, três $(0,2 \%)$ pacientes apresentaram insuficiência renal, seis $(0,4 \%)$ tiveram acidente vascular cerebral, $19(1,3 \%)$ foram reoperados por sangramento, $19(1,3 \%)$ tiveram mediastinite, $18(1,25 \%)$ infarto agudo do miocárdio e $212(14,7 \%)$ apresentaram fibrilação atrial. Houve 50 (3,5\%) óbitos, sendo $29(2,5 \%)$ entre 1148 pacientes operados eletivamente, nove $(4,7 \%)$ entre 190 pacientes submetidos a reoperação coronariana e $12(11,7 \%)$ entre 102 pacientes operados em caráter de emergência.

Conclusão: Com a evolução da tecnologia biomédica, todos os vasos do coração passaram a ser abordados. Estes dados sugerem que a operação para revascularização do miocárdio é segura e eficaz, podendo ser aplicada em todos os pacientes que necessitem de cirurgia coronariana com baixos índices de complicações e mortalidade.

Descritores: Revascularização miocárdica, métodos. Circulação extracorpórea. Ponte de artéria coronária.

Trabalho realizado no Serviço de Cirurgia Cardiovascular - Santa Casa de Misericórdia - PUC/ PR

Endereço para correspondência: Dr. Rodrigo M Milani

Serviço de Cirurgia Cardíaca.

Santa Casa de Misericórdia - PUC/PR

Praça Rui Barbosa, 694 Centro -Curitiba,Paraná

CEP: $80010-030$

Tel: (41) 323-4926. Fax: (41) 232-8982

Artigo recebido em maio de 2004

E-mail: rodrigo.milani@sbccv.org.br 


\begin{abstract}
Objective: To evaluate the immediate postoperative results of off-pump myocardium revascularization surgery by analyzing complications and mortality.

Method: A total of 1440 patients were submitted to offpump myocardium revascularization. The surgical technique is consisted in proximal occlusion of the approached artery, the application of the LIMA graft in the posterior pericardial deflection and stabilization of the target artery with a suction stabilizer. The distal anastomoses were performed first.

Results: Among the patients evaluated, 924 were male and 516 were female,with a mean age of $63.12 \pm 8.76$ years. The ejection fraction was normal in 749 patients, 740 (51.4\%) patients had suffered previous myocardial infarction and 687 $\mathbf{4 7 . 6 \% )}$ patients were in functional class III or IV. The mean EuroSCORE was $4.93 \pm 3.32$. The mean number of dial anastomoses was $3.12 \pm 1.23$ per patient. A total of $1173(81.5 \%)$ patients remained less than 12 hours on mechanical ventilation and among them, $888(61.7 \%)$ remained for less than six hours. The stay in the ICU was of one night for 330
\end{abstract} $(22.8 \%)$ patients, for two nights for $930(64.6 \%)$ patients and

\section{INTRODUÇÃO}

O uso da circulação extracorpórea em pacientes submetidos a operações coronarianas é comumente associado a um aumento de morbidade no período pósoperatório, devido à reação inflamatória sistêmica por ela ocasionada [1]

Inúmeros artigos, publicados ao longo dos últimos anos, associaram a circulação extracorpórea a uma série de complicações observadas em pacientes operados para revascularização do miocárdio [2-4]. Este fato fez com que as operações coronarianas sem circulação extracorpórea, após os trabalhos pioneiros de BUFFOLO et al. [5] e BENETTI [6], ganhassem interesse mundial, com estudos de diversos centros [7-12] sendo apresentados no Brasil e no exterior, demonstrando a eficiência e segurança deste tipo de operação, que cada vez mais ganha novos adeptos entre os cirurgiões cardiovasculares, cardiologistas clínicos e intensivistas.

No presente estudo, apresentamos uma análise retrospectiva dos primeiros 1440 pacientes submetidos à operação para revascularização do miocárdio sem circulação extracorpórea, nos últimos cinco anos.

Em 1999, ano em que iniciamos o programa, pouco mais de $50 \%$ dos pacientes coronarianos foram submetidos à operação sem circulação extracorpórea. Com a familiarização da técnica e acúmulo de experiência, este número subiu para $100 \%$ no último ano, mostrando que todos os pacientes que necessitam de operação para revascularização do miocárdio
$182(12.4 \%)$ patients remained three or more nights. In regards to complications, three $(0.2 \%)$ patients presented with renal insufficiency, six $(0.4 \%)$ patients suffered stroke, 19 $(1.3 \%)$ patients were reoperated for bleeding, $19(1.3 \%)$ patients had mediastinitis, $18(1.25 \%)$ patients suffered severe myocardial infarction and $212(14.7 \%)$ presented atria fibrillation. There were $50(3.5 \%)$ deaths, $29(2.5 \%)$ of them being among the 1148 operated electively, nine (4.7\%) among 190 patients submitted to coronary re-operation and 12 $(11.7 \%)$ among 102 patients undergoing emergency operations.

Conclusion: With the evolution of biomedical technological, all the vessels of the heart are now approached. These data suggest that the operation for myocardial revascularization is safe and efficient. It can be applied to all patients who need coronary surgery, with low rates of complication and mortality.

Descriptors: Myocardial revascularization, methods. Extracorporeal circulation. Coronary artery bypass.

podem ser beneficiados com a técnica sem circulação extracorpórea.

\section{MÉTODO}

Entre janeiro de 1999 e janeiro de 2004, 1440 pacientes, com idade média de 63,12 $\pm 8,76$ anos, variando de 28 a 94 anos foram submetidos à operação para revascularização do miocárdio sem circulação extracorpórea. Deste total, 183 pacientes tinham idade superior a 75 anos. Quanto ao sexo, 924 pacientes eram do sexo masculino e 516 ao feminino.

A partir de setembro de 2001, todos os pacientes tiveram seus dados armazenados pelo banco de dados utilizados pela Society of Thoracic Surgeons [13] e pelo índice de risco desenvolvido pela European Association for Cardiothoracic Surgery, o EuroSCORE [14,15]. Os pacientes operados antes desta data tiveram seus dados coletados por meio da análise dos prontuários.

Todos os pacientes foram operados por toracotomia mediana. A técnica anestésica utilizada, bem como a maneira usada para obtenção dos enxertos, foi previamente descrita [16]. Ao término da obtenção dos enxertos, o paciente foi heparinizado com uma dose de $2,5 \mathrm{mg} / \mathrm{kg}$. Em seguida, um ponto de Ethibond 2-0, fixado à porção central de uma fita medindo $80 \mathrm{~cm}$ de comprimento por $3 \mathrm{~cm}$ de largura foi aplicado na deflexão pericárdica posterior, entre as veias cava inferior e pulmonar inferior esquerda [16]. Este ponto permitia que, com a mobilização da fita, todas as regiões do coração fossem expostas, causando pouca variação 
hemodinâmica. A mesa de operação foi posicionada em Trendlemburg e lateral esquerdo. Nesta etapa, a pressão venosa central foi mantida acima de $15 \mathrm{mmHg}$.

As anastomoses distais foram rotineiramente feitas inicialmente, começando-se sempre que possível pela artéria coronária direita e seus ramos, seguindo-se pelos ramos marginais da artéria circunflexa, ramos diagonalis, ramos diagonais e, por fim, ramo descendente anterior. Nos casos em que uma área parecia mais isquêmica que as demais, esta região revascularizada inicialmente. A artéria abordada foi ocluída temporariamente, em sua porção proximal, com um ponto de prolene 4-0 ancorado em uma pequena tira de silicone. A área em que se trabalhava foi fixada com auxílio de estabilizador por sucção (Octopus System, Medtronic Corporation). A anastomose distal foi, então, realizada com sutura contínua de prolene 7-0. Ao término destas, a pressão sistólica foi mantida em $100 \mathrm{mmHg}$ e as anastomoses proximais feitas com sutura contínua de prolene 6-0. Liberado o pinçamento parcial da aorta, $2 \mathrm{mg} / \mathrm{kg}$ de heparina foram revertidos com sulfato de protamina (dose inicial de heparinização foi de $2,5 \mathrm{mg} / \mathrm{kg}$ ) e a operação finalizada. Nos últimos seis meses, deu-se preferência para que, sempre que possível, o paciente fosse acordado e extubado na sala de operação.

Em relação a fatores de risco para doença coronária, 350 eram diabéticos, 572 dislipidêmicos, 921 tinham história de tabagismo e 1197 eram hipertensos. Em 208 pacientes não se detectou nenhum fator de risco coronariano.

A maioria dos pacientes encontrava-se em classe funcional II (538), com 215 pacientes em classe I, $401 \mathrm{em}$ classe III e 286 em classe IV. Setecentos e quarenta pacientes tinham antecedente de infarto do miocárdio, com 115 apresentando quadro de infarto em um período inferior a dez dias da data da operação. A fração de ejeção encontrada foi acima de 50\% em 749 pacientes, entre 30 e $50 \%$ em 511 e abaixo de 30\% em 180 .

Em relação a doenças associadas (Tabela 1), seis pacientes eram portadores de marcapasso cardíaco, 23 tinham insuficiência renal crônica, 15 tinham doença gastrointestinal, 27 eram portadores de fibrilação atrial crônica, 44 tinham doença arterial vascular periférica, 45 apresentavam antecedente de acidente vascular cerebral e 68 eram portadores de DPOC grave.

Tabela 1. Doenças associadas.

\begin{tabular}{lll}
\hline BAVT & 6 & $0,4 \%$ \\
Insuficiência renal & 23 & $1,6 \%$ \\
Doença gastrointestinal & 15 & $1,0 \%$ \\
Fibrilação atrial & 27 & $1,8 \%$ \\
Doença arterial vascular periférica & 44 & $3,0 \%$ \\
Acidente vascular cerebral & 45 & $3,0 \%$ \\
DPOC & 68 & $4,7 \%$ \\
\hline
\end{tabular}

DPOC - Doença Pulmonar Obstrutiva Crônica
A cineangiocoronariografia revelou doença em um único vaso em 73 pacientes, doença em dois vasos em 384, e em três ou mais vasos em 983 pacientes. A lesão de tronco de artéria coronária esquerda estava presente em 132 pacientes.

Cento e sessenta e sete pacientes haviam sido submetidos à operação para revascularização do miocárdio prévia, enquanto 347 tinham antecedente de angioplastia.

No período pré-operatório imediato, 303 pacientes estavam em uso de nitroglicerina endovenosa para controle de angina, 52 estavam usando drogas vasoativas devido à hipotensão e 21 doentes necessitaram de balão intraórtico antes do início da operação. Cento e dois pacientes foram operados em caráter de emergência.

A pontuação média obtida no EuroSCORE foi de $4,93 \pm 3,32$, variando de 0 a 18 pontos.

\section{RESULTADOS}

O número total de anastomoses distais foi de 4993, com média de $3,12 \pm 1,23$ por paciente, variando de uma a sete anastomoses. A artéria torácica interna esquerda foi utilizada em 1185 pacientes e em 293 foram utilizados apenas enxertos arteriais.

O tempo de ventilação mecânica variou de zero a 288 horas. Cento e dezenove pacientes foram extubados na sala de operação e 1173 pacientes permaneceram menos de 12 horas em ventilação mecânica, sendo destes, 890 permaneceram entubados por um período inferior a 6 horas. A permanência em unidade de terapia intensiva foi inferior a duas noites em 1260 pacientes, com 330 destes permanecendo apenas uma noite na unidade.

Em relação a complicações observadas no período pós-operatório (Tabela 2), três pacientes desenvolveram insuficiência renal aguda, seis apresentaram quadro de acidente vascular cerebral, 19 necessitaram de reoperação por sangramento, 19 desenvolveram mediastinite, 18 apresentaram quadro de infarto agudo do miocárdio e 212 desenvolveram fibrilação atrial. Sete pacientes operados no início da série precisaram ser reoperados em um período inferior a 12 meses da primeira operação.

Tabela 2. Complicações pós-operatórias.

\begin{tabular}{lll}
\hline Insuficiência renal & 3 & $0,2 \%$ \\
Acidente vascular cerebral & 6 & $0,4 \%$ \\
Reoperação precoce & 7 & $0,5 \%$ \\
Reoperação por sangramento & 19 & $1,3 \%$ \\
Mediastinite & 19 & $1,3 \%$ \\
Infarto do miocárdio & 18 & $1,3 \%$ \\
Fibrilação atrial & 212 & $14,7 \%$ \\
\hline
\end{tabular}


O número total de óbitos foi de $50(3,5 \%), \operatorname{com} 29(2,5 \%)$ mortes ocorrendo entre 1148 pacientes operados eletivamente, nove $(4,7 \%)$ óbitos entre 190 pacientes submetidos a reoperação coronariana e $12(11,7 \%)$ entre 102 doentes operados em situação de emergência. A Tabela 3 mostra as principais causas de óbito.

Tabela 3. Causas de óbito.

\begin{tabular}{lll}
\hline Insuficiência renal & 2 & $0,1 \%$ \\
Acidente vascular cerebral & 2 & $0,1 \%$ \\
Causas metabólicas & 4 & $0,2 \%$ \\
Tromboembolismo pulmonar & 4 & $0,2 \%$ \\
Sangramento & 6 & $0,4 \%$ \\
Mediastinite & 9 & $0,6 \%$ \\
Causa desconhecida & 9 & $0,5 \%$ \\
Choque cardiogênico & 14 & $1,0 \%$ \\
\hline
\end{tabular}

\section{COMENTÁRIOS}

A operação para revascularização do miocárdio com auxílio da circulação extracorpórea é considerada um procedimento "Gold Standard" devido aos seus excelentes resultados e a sua reprodutibilidade nos mais diversos centros. Apesar de ótimos resultados em até 15 anos de follow up [17], inúmeras publicações têm demonstrado os efeitos deletérios da circulação extracorpórea[1-4].

Nos últimos anos, após a melhora nas técnicas de estabilização e exposição do coração [18], o interesse pela revascularização do miocárdio cresceu imensamente, como pode ser visto pelos trabalhos apresentados pelos mais diversos centros [7-12]. A contribuição brasileira foi fundamental para este aumento de interesse, inicialmente com BUFFOLO et al. [5] e, posteriormente, com LIMA[18] e RIVETTI \& GANDRA[19].

A revascularização do miocárdio com o coração em sua atividade normal é associada à diminuição da microembolia cerebral e da resposta inflamatória e à maior proteção renal $[1,20,21]$. Além disto, resultados superiores ao da técnica convencional têm sido apresentados em subgrupos de pacientes com maior risco para a operação, como os pacientes com importante disfunção ventricular [22] e os pacientes submetidos a reoperações coronarianas [23].

Em nossa série, analisamos os resultados imediatos dos primeiros 1440 pacientes operados sem circulação extracorpórea em nossa instituição. Neste grupo, apenas nos dois primeiros anos de experiência alguns pacientes foram selecionados para operação sem CEC, sendo que nos dois últimos anos, praticamente todos os pacientes foram operados desta maneira. No último ano, o único paciente operado com auxílio da circulação extracorpórea estava sendo submetido à terceira operação coronariana e, durante a toracotomia, houve grande lesão de ventrículo direito, sendo necessária instalação da CEC para resolução satisfatória do problema. Todos os demais foram operados sem CEC, mostrando que atualmente todos os vasos do coração podem ser abordados de maneira adequada com esta técnica.

A incidência de complicações observadas em pacientes operados sem circulação extracorpórea foi baixa em nossa série, como por exemplo, insuficiência renal $(0,2 \%)$ e acidente vascular cerebral $(0,4 \%)$. Outras complicações como mediastinite e infarto do miocárdio também tiveram baixa incidência, sendo que em relação ao infarto a taxa apresentou decréscimo importante nos últimos casos.

Outro fator importante a ser considerado, a reoperação precoce, visto que a qualidade das anastomoses sempre foi questionada em relação as operações sem circulação extracorpórea, também teve baixa incidência $(0,51 \%)$ e todos os casos são pacientes do início da experiência.

$\mathrm{O}$ segundo questionamento feito pelos críticos das operações sem CEC é o baixo número de anastomoses distais por paciente. Em nossa série total, o número médio de anastomoses distais por paciente foi de $3,12 \pm 1,23$, com este número subindo para 3,22 nos últimos 12 meses, mostrando que a operação sem CEC permite a revascularização total do miocárdio.

\section{CONCLUSÃO}

Concluindo, o grande avanço da indústria biomédica na área das operações menos invasivas tornou possível a revascularização de todos os vasos do miocárdio. Em uma fase inicial da experiência, é esperada a ocorrência de arritmias e instabilidade hemodinâmica até para confecção de anastomoses na parede anterior, o que, com a experiência, raramente acontece mesmo quando se faz necessário colocar três ou quatro enxertos nas paredes lateral e posterior do coração. Estudos sugerem um benefício grande das operações sem circulação extracorpórea sobre as convencionais [24-26], já sendo demonstrado bons resultados em médio prazo [27-29]. Com o avanço cada vez mais intenso da cardiologia intervencionista, o cirurgião cardíaco vem sendo cobrado a apresentar operações menos invasivas e com excelentes resultados, o que, a nosso ver, inicia-se pelas operações sem CEC. Os dados da série apresentados mostram que as operações para revascularização do miocárdio sem circulação extracorpórea são seguras e eficazes, apresentando baixo índice de complicações e de mortalidade, permitindo a realização de anastomoses de boa qualidade, bem como a revascularização total do miocárdio. Atualmente, é o método de escolha em nossa instituição para as operações coronarianas. 


\section{REFERÊNCIAS BIBLIOGRÁFICAS}

1. Matata BM, Sosnowski AW, Galinanes M. Coronary artery bypass graft surgery off-pump on the beating heart abolishes inflammation and oxidative stress. Artif Organ. 1999;23:662-3.

2. Kirklin JK. Prospects for understanding and eliminating the deleterious effects of cardiopulmonary bypass. Ann Thorac Surg. 1991;51(4):529-31.

3. Brasil LA, Gomes WJ, Salomão R, Buffolo E. Inflammatory response after myocardial revascularization with or without cardiopulmonary bypass. Ann Thorac Surg. 1998;66(1):56-9.

4. Ascione R, Lloyd CT, Lotto AA, Pitsis AA, Angelini GD. Inflammatory response after coronary revascularization with or without cardiopulmonary bypass. Ann Thorac Surg. 2000;69(4):1198-204

5. Buffolo E, Andrade JCS, Branco JN, Teles CA, Aguiar LF, Gomes WJ. Coronary artery bypass grafting without cardiopulmonary bypass. Ann Thorac Surg. 1996;61(1):63-6.

6. Benetti FJ. Direct coronary surgery with saphenous vein bypass without either cardiopulmonary bypass or cardiac arrest. J Cardiovasc Surg. 1985;26(3):217-22.

7. Mack MJ. Beating heart surgery for coronary revascularization: is it the most important development since the introduction of the heart-lung machine? Ann Thorac Surg. 2000;70(5):1774-8.

8. Milani RM, Brofman PRS, Moutinho JA. Morbidity and mortality impact in the myocardial revascularization without extracorporeal circulation. In: 12th World Congress on Cardiothoracic Surgery. Luzern, Switzerland;2002.

9. Moshkovitz Y, Lusky A, Mohr R. Coronary artery bypass without cardiopulmonary bypass: analysis of short-term and mid-term outcome in 220 patients. J Thorac Cardiovasc Surg. 1995;110(4 Pt 1):979-87.

10. Patel NC, Grayson AD, Jackson M, Au J, Yonan N, Hasan R et al. The effect off-pump coronary artery bypass surgery on in-hospital mortality and morbidity. Eur J Cardiothorac Surg. 2002;22(2):255-60

11. Pinheiro BB, Fagundes WV, Ramos MC, Azevedo VLB, Silva JM. Revascularização do miocárdio sem circulação extracorpórea em pacientes multiarteriais: experiência de 250 casos. Rev Bras Cir Cardiovasc. 2002;17(3):242-7.

12. Lobo Filho JG, Leitão MCA, Lobo Filho HG, Soares JPH, Magalhães GA, Leão Filho CSC et al. Cirurgia de revascularização coronariana esquerda sem CEC e sem manuseio da aorta em pacientes acima de 75 anos: Análise das mortalidades imediata e a médio prazo e das complicações neurológicas no pós-operatório imediato. Rev Bras Cir Cardiovasc. 2002;17(3):208-14
13. Clark RE. The STS Cardiac Surgery National Database: an update. Ann Thorac Surg. 1995;59(6):1376-81.

14. Roques F, Nashef SA, Michel P, Gauducheau E, de Vincentiis $\mathrm{C}$, Baudet $\mathrm{E}$ et al. Risk factors and outcome in European cardiac surgery: analysis of the EuroSCORE multinational database of 19030 patients. Eur J Cardiothorac Surg. 1999;15(6):816-23.

15. Nashef SA, Roques F, Michel P, Gauducheau E, Lemeshow S, Salamon R. European System for Cardiac Operative Risk Evaluation (EuroSCORE). Eur J Cardiothorac Surg. 1999;16(1):9-13.

16. Milani RM. Análise dos resultados imediatos da operação para revascularização do miocárdio sem pinçamento total da aorta [Tese de mestrado]. Curitiba:Universidade Federal do Paraná, 2000.

17. Fiore AC, Naunhein KS, McBride LR, Peigh PS, Pennington DG, Kaiser GC et al. Fifteen-year follow-up for double internal thoracic artery grafts. Eur J Cardiothorac Surg. 1991;5(5):248-52.

18. Lima RC. Padronização técnica de revascularização miocárdica da artéria circunflexa e seus ramos sem circulação extracorpórea [Tese de doutorado]. São Paulo: Universidade Federal de São Paulo. Escola Paulista de Medicina, 1999.

19. Rivetti LA, Gandra SM. Initial experience using an intraluminal shunt during revascularization of the beating heart. Ann Thorac Surg. 1997;63(6):1742-7.

20. Bowles BJ, Lee JD, Dang CR, Taoka SN, Johnson EW, Lau EM et al. Coronary artery bypass performed without the use of cardiopulmonary bypass is associated with reduced cerebral microemboli and improved clinical results. Chest. 2001;119(1):25-30.

21. Tang AT, Knott J, Nanson J, Hsu J, Haw MP, Ohri SK. A prospective randomized study to evaluate the renoprotective action of beating heart coronary surgery in low risk patients. Eur J Cardiothorac Surg. 2002;22(1):118-23.

22. Eryilmaz S, Corapcioglu T, Eren NT, Yazicioglu L, Kaya K Akalin H. Off-pump coronary artery bypass surgery in the left ventricular dysfunction. Eur J Cardiothorac Surg. 2002;21(1):36-40.

23. Stamou SC, Pfister AJ, Dangas G, Dullum MK, Boyce SW, Bafi AS et al. Beating heart versus conventional single-vessel reoperative coronary artery bypass. Ann Thorac Surg. 2000;69(5):1383-7.

24. Racz MJ, Hannan EL, Isom OW, Subramanian VA, Jones RH, Gold JP et al. A comparison of short and long-term outcomes after off-pump and on-pump coronary artery bypass graft surgery with sternotomy. J Am Coll Cardiol. 2004:43(4):557-64. 
25. Gerola LR, Buffolo E, Jazbik W, Botelho B, Bosco J, Brasil LA et al. Off-pump versus on-pump myocardial revascularization in low-risk patients with one or two vessel disease: perioperative results in a multicenter randomized controlled trial. Ann Thorac Surg. 2004;77(2):569-73.

26. Mack MJ, Pfister A, Bachand D, Emery R, Magee MJ, Connolly $\mathrm{M}$ et al. Comparison of coronary bypass surgery with and without cardiopulmonary bypass in patients with multivessel disease. J Thorac Cardiovasc Surg. 2004;127(1):167-73.
27. Reston JT, Tregear SJ, Turkelson CM. Meta-analysis of shortterm and mid-term outcomes following off-pump coronary artery bypass grafting. Ann Thorac Surg. 2003;76(5):1510-5.

28. Lima RC, Escobar MAS, Lobo Filho JG, Diniz R, Saraiva A, Césio A et al. Resultados cirúrgicos na revascularização do miocárdio sem circulação extracorpórea: análise de 3410 pacientes. Rev Bras Cir Cardiovasc. 2003;18(3):261-7.

29. Aguiar LF, Andrade JCS, Branco JN, Palma JH, Teles CA, Gerola LR et al. Revascularização do miocárdio sem circulação extracorpórea: resultados da experiência de 18 anos de sua utilização. Rev Bras Cir Cardiovasc. 2001;16(1):1-6. 